\title{
Prediciendo la distribución de Polylepis: bosques Andinos vulnerables y cada vez más importantes
}

\author{
Predicting Polylepis distribution: vulnerable and increasingly important \\ Andean woodlands
}

\author{
Brian R. Zutta ${ }^{1,2}$, Phillip W. Rundel ${ }^{1}$, Sassan Saatchi' ${ }^{2}$, Jorge D. Casana ${ }^{3}$, Paul Gau- \\ thier $^{4}$, Aldo Soto ${ }^{5}$, Yessenia Velazco ${ }^{6}$, y Wolfgang Buermann ${ }^{7}$
}

1 Center for Embedded Networked Sensing, University of California Los Angeles, 3563 Boelter Hall, Los Angeles, CA 90095-1596, EEUU. Email Brian R. Zutta:

bzutta@ucla.edu

Email Phillip W. Rundel:

rundel@lifesci.ucla du

2 Jet Propulsion Laboratory, California Institute of Technology, 4800 Oak Grove Drive, Pasadena, CA 91109, EEUU.

saatchi@congo.jpl.nasa.gov

3 Parque Nacional Huascarán, Instituto Nacional de Recursos Naturales, Federico Salyrosas $\mathrm{N}^{\circ}$ 555, Huaraz, Perú.

4 Research School of Biology. The Australian National University, Canberra, ACT 0200. Austra paul.gauthier@anu.edu.au

5 Centro de Datos para la Conservación, Universidad Naciona servación, Universidad Nacional Agraria La Molina, Av. La Moli aldo_soto@lamolina.edu.pe 6 Department of Biological Sciences, California State University Los Angeles, 5151 State University Anges, 5 I S ve, LosAngels, CA90032, EEUU. yvelazc@calstatela.edu

7 Center for Tropical Research, Institute of the Environment, University of California Los Angeles, La Kretz Hall, Suite 300 , La Kretz Hall, Suite 300, Box 1496. EEUU.

buermann@ucla.edu

Presentado: 23/05/2012 Aceptado: $\quad$ 23/08/2012 Publicado online: 13/11/2012

\section{Resumen}

Los bosques de Polylepis son recursos vitales para la conservación de la biodiversidad y funciones hidrológicas, la cual se verá alterada por el cambio climático a nivel mundial desafiando la sostenibilidad de las comunidades locales. Sin embargo, estos ecosistemas andinos de gran altitud son cada vez más vulnerables debido a la presión antropogénica como la fragmentación, deforestación y el incremento en el ganado. La importancia para predecir la distribución de bosques nativos ha aumentado para contrarrestar los efectos negativos del cambio climático a través de la conservación y la reforestación. El objetivo de este estudio fue desarrollar y analizar los modelos de distribución de dos especies, Polylepis sericea y $P$. besseri, que forman bosques extensos a lo largo de los Andes. Este estudio utilizó el programa MAXENT, el clima y capas ambientales de una resolución de $1 \mathrm{Km}$. El modelo de distribución previsto para $P$. sericea indica que la especie podría estar situada en una variedad de hábitats a lo largo de la Cordillera de los Andes, mientras que $P$. besseri se limitaba a las grandes alturas del sur de Perú y Bolivia. Para ambas especies, los metros de elevación y la temperatura son los factores más importantes para la distribución prevista. El perfeccionamiento del modelo de Polylepis y otras especies andinas utilizando datos de satélites cada vez más disponibles al público demuestran el potencial para ayudar a definir las áreas de diversidad y mejorar las estrategias de conservación en los Andes.

Palabras claves: distribución, MAXENT, MODIS, Polylepis, sensores remotos, QSCAT, WorldClim.

\section{Abstract}

Polylepis woodlands are a vital resource for preserving biodiversity and hydrological functions, which will be altered by climate change and challenge the sustainability of local human communities. However, these highaltitude Andean ecosystems are becoming increasingly vulnerable due to anthropogenic pressure including fragmentation, deforestation and the increase in livestock. Predicting the distribution of native woodlands has become increasingly important to counteract the negative effects of climate change through reforestation and conservation. The objective of this study was to develop and analyze the distribution models of two species that form extensive woodlands along the Andes, namely Polylepis sericea and P. weberbaueri. This study utilized the program MAXENT, climate and remotely sensed environmental layers at $1 \mathrm{Km}$ resolution. The predicted distribution model for $P$. sericea indicated that the species could be located in a variety of habitats along the Andean Cordillera, while $P$. weberbaueri was restricted to the high elevations of southern Peru and Bolivia. For both species, elevation and temperature metrics were the most significant factors for predicted distribution. Further model refinement of Polylepis and other Andean species using increasingly available satellite data demonstrate the potential to help define areas of diversity and improve conservation strategies for the Andes.

Keywords: MAXENT, MODIS, Polylepis, QSCAT, remote sensing, species distribution modeling, WorldClim.

\section{Introducción}

Cada vez más vulnerables, los bosques de Polylepis (Rosaceae) son un ecosistema Andino importante para la protección de la biodiversidad y la mitigación de los efectos del cambio climático local. El género Polylepis incluye aproximadamente 27 especies (Mendoza \& Cano 2011, Kessler \& Schmidt-Lebuhn 2006) que forman bosques de hoja perenne con poblaciones muy fragmentadas a lo largo de las alturas de los Andes tropicales y subtropicales. Se estima que quedan menos del 10\% de su extensión original en las regiones altas de Bolivia y Perú (Fjeldså \& Kessler 1996) y catorce especies están listados como vulnerables (IUCN 2011). Sin embargo, estos bosques contienen una variedad de especies endémicas y en peligro de extinción (Servat et al. 2002) y cumplen funciones importantes hidrológicas en intercepción de neblina (Fig. 1; Fjeldså 2002).

Todas las especies de Polylepis son arbustos o árboles, algunos hasta $10 \mathrm{~m}$ de altura y comparten características morfológicas, que incluyen troncos rojos y torcidos, corteza delgada y exfoliante, y pequeñas hojas imparipinnadas. Varias especies son

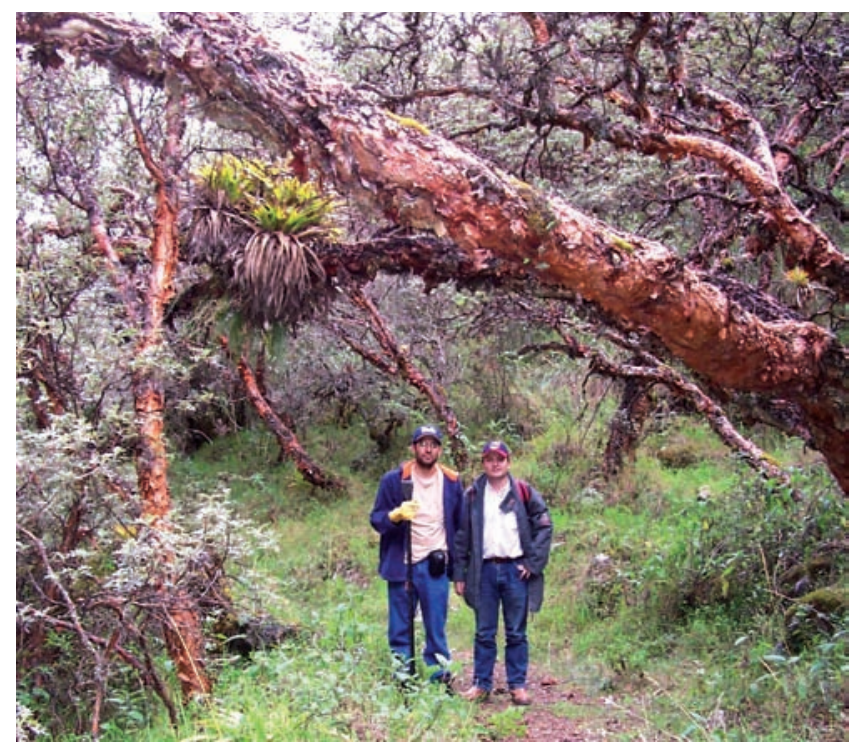

Figura 1. Mezcla de bosques de Polylepis en el Parque Nacional Huascarán, Cordillera Blanca del Perú, que soporta una variedad de epífitas y es importante para la intercepción de neblina. Foto por Autor. 
Tabla 1. Descripción de la distribución de especies de Polylepis, notas sobre la ecología y estado de conservación*.

\begin{tabular}{|c|c|c|}
\hline Especie & Distribución & Notas sobre ecología \\
\hline P. australis & Central y nor-oeste de Argentina ${ }^{(e)}$ & Alto bosque nublado, restringida a laderas y barrancos. \\
\hline P. besseri & Sur Perú a Bolivia & De 3000-4100 m en algunas áreas a menudo se forman bosques puros. \\
\hline P. canoi & Ayacucho, Cusco y Junín, Perú(e) & De 3350-3400. \\
\hline P. crista-galli & Sur de Bolivia a norte de Argentina(v) & $\begin{array}{l}\text { Por debajo de } 3000 \text { a } 3900 \mathrm{~m} \text {, favorece laderas secas en las elevaciones } \\
\text { más bajas. }\end{array}$ \\
\hline P. flavipila & Andina occidental del centro del Perú(e) & Pendientes áridas de 3600-4100 m. \\
\hline P. hieronymi & Sur de Bolivia hasta el norte de Argentina ${ }^{(v)}$ & $\begin{array}{l}\text { Especie pionera que se encuentra por debajo } 3000-3400 \mathrm{~m} \text { y laderas } \\
\text { orientales. }\end{array}$ \\
\hline P. incana & Ecuador hasta el sur de Perú(v) & $\begin{array}{l}\text { Laderas occidentales de Ecuador desde 3500-4100 m. Hasta } 4200 \text { m en } \\
\text { Perú. }\end{array}$ \\
\hline P. incarum & Sur Peruano a Bolivia & Principalmente en 3800-4200 m en zonas con lluvias estacionales. \\
\hline P. lanata & Sur Peruano a Bolivia & Pendientes envueltas en neblina de $2900-4100 \mathrm{~m}$. \\
\hline P. lanuginosa & Ecuador $^{(e, v)}$ & Limitados a ambientes áridos de montaña. \\
\hline P. microphylla & Ecuador y aislado en Perú(v) & $\begin{array}{l}\text { Aislado en el Volcán Chimborazo, Ecuador y trasplantes precolombinos } \\
\text { en Perú. }\end{array}$ \\
\hline P. multijuga & Norte del Peru ${ }^{(e, v)}$ & Bosques montañosos bajos. \\
\hline P. neglecta & Central y sur de Bolivia(e,v) & $\begin{array}{l}\text { Encontrado en } 2400-3500 \mathrm{~m} \text { y tiende a crecer en arroyos o en lomas } \\
\text { expuestas. }\end{array}$ \\
\hline P. pacensis & 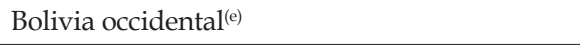 & Valles inter-andinos y cordilleras occidentales desde $3200-4100$ m. \\
\hline P. pauta & Ecuador y aislado en Perú y Bolivia(v) & Bosques de montaña hasta 4000 m, en zonas con neblina frecuentes. \\
\hline P. pepei & Peru central hasta el noreste de Bolivia(v) & $\begin{array}{l}\text { En la zona de transición de los bosques montañosos húmedos a pajo- } \\
\text { nales de puna. }\end{array}$ \\
\hline P. quadrijuga & Cordilleras orientales de Colombia ${ }^{(e)}$ & Por encima de la línea de bosque continuo hasta el páramo. \\
\hline P. racemosa & Perú a Bolivia, y aislado en Ecuador(v) & $\begin{array}{l}\text { Límite superior de bosques montañosos húmedos y zonas de niebla } \\
\text { frecuentes. }\end{array}$ \\
\hline P. reticulata & Ecuador y aislado en el norte y centro del Perú(v) & $\begin{array}{l}\text { Se encuentran en los bosques nublados altos y algunos valles inter- } \\
\text { andinos. }\end{array}$ \\
\hline P. rugulosa & Suroeste de Perú a extremo norte de Chile ${ }^{(v)}$ & Entre $3000-4600$ m por cañón y laderas orientados sudoeste. \\
\hline P. sericea ${ }^{* *}$ & Venezuela a Bolivia & $\begin{array}{l}\text { Bosques húmedos de montaña, de } 2600 \text { m hasta } 4100 \text { m en muchas } \\
\text { zonas del Perú. }\end{array}$ \\
\hline P. subsericans & Centro y sur de Perú(e,v) & Encontrado en $2900-5100$ m, a menudo en las partes altas de cañones. \\
\hline P. subtusalbida & Sur de Perú y Bolivia central ${ }^{(\mathrm{e})}$ & $\begin{array}{l}\text { En las regiones con una precipitación anual de } 600 \mathrm{~mm} \text { y una altura de } \\
3000-4500 \mathrm{~m} \text {. }\end{array}$ \\
\hline P. tarapacana & Frontera de Argentina, Bolivia, Chile, y Perú ${ }^{(c)}$ & Se encuentran en alturas extremas de 4000-5200 m y paisajes áridos. \\
\hline P. tomentella & Bolivia a Argentina, y aislado en Perú central & $\begin{array}{l}\text { Ocupan una variedad de hábitats de mucha altitud con precipitaciones } \\
\text { de } 450-800 \mathrm{~mm} \text {. }\end{array}$ \\
\hline P. triacontandra & Sur de Perú a Bolivia, aislado en centro de Perú & $\begin{array}{l}\text { Altos límites de los bosques húmedos, a } 4000 \text { m en regiones con niebla } \\
\text { frecuente. }\end{array}$ \\
\hline P. weberbaueri** & Ecuador hasta el sur de Perú(v) & Los bosques húmedos montañosos, de 2500-4300 m. \\
\hline
\end{tabular}

* De acuerdo al 2011 Lista Roja de Especies Amenazadas.

** Especies modelado.

(e) Endémico, (v) Vulnerable, (c) Casi amenazada

conocidas por particulares características ecológicas dentro de su distribución en los Andes (Tabla 1). Por ejemplo, P. pauta se puede encontrar en ceja de selva en Ecuador y grupos aislados en el sur Peruano (Cierjacks et al. 2008). Polylepis tarapacana es conocida por alcanzar altitudes hasta $5200 \mathrm{~m}$ en el Volcán Sajama en Bolivia, que puede ser la elevación más alta para las plantas leñosas en el mundo (Garcia-Nuñez et al. 2004). Polylepis sericea es la única especie que se encuentra en Venezuela y tiene la distribución más amplia del género, alcanzando hasta el norte de Bolivia (Azócar et al. 2007).

La distribución natural de los bosques de Polylepis ha sido debatida durante mucho tiempo como consecuencia de la actual fragmentación potencialmente resultante de presión de miles de años de poblaciones humanas en los Andes (Kessler 2002). Durante los años, la hipótesis alternativas han sugerido que los bosques de Polylepis originalmente cubría la mayor parte de las montañas andinas (Ellenberg 1979) o habían rangos limitados por microclimas específicos (Simpson 1986). La reconstrucción de paleo bosques de Polylepis ha sido difícil, ya que el polen fósil es casi idéntico a la del género Acaena (Rosaceae) (Kessler 2002). Aunque el polen en elevaciones por encima de $4800 \mathrm{~m}$ es más probable que sea de Polylepis en lugar de Acaena que es principalmente restringido a bosques nublados (Hanselman et al. 2005), los paleo bosques son muchas veces identificados como Polylepis/Acaena (Hanselman et al. 2005; Gosling et al. 2008). Hoy en día, estos bosques enfrentan una presión de pastoreo de ganado (Teich et al. 2005), fuego (Cierjacks et al. 2008) y la expansión de carreteras (Purcell \& Brelsford 2004), todos los cuales han reducido la extensión de hábitat y su integridad (Jameson \& Ramsay 2007). Complicando las evaluaciones de conservación, se han presentado las similitudes morfológicas de muchas especies de Polylepis que han llevado a muchos estudios para agruparlos como un único tipo de bosque (Simpson 1986; Kessler \& Schmidt-Lebuhn 2006). Los Modelos de distribución son una técnica que mejora continuamente para estimar el 
potencial de una especie que ocurren en zonas que no han sido previamente estudiados (Guisan \& Thuiller 2005; Hernandez et al. 2008). Las estrategias de conservación frecuentemente utilizan modelos para dirigir estudios sobre la ubicación de especies (Raxworthy et al. 2003; Bourg et al. 2005; Hernandez et al. 2008) e identificar áreas prioritarias para la conservación (Wilson et al. 2005; Hernandez et al. 2008). Estudios recientes utilizando modelos de distribución tocan estos temas para la conservación de los hábitats amenazados (Riordan \& Rundel 2009) y ayudar a vincular la distribución genética y geográfica (Thomassen et al. 2011). En general, mucho de estos modelos solo requieren datos de localización y datos ambientales para realizar un análisis estadístico que establezca las relaciones especie-medio ambiente y construir un modelo espacial de ocurrencia.

Cada vez más disponibles, imágenes de satélites y relativamente rápidos modelos de análisis de distribución pueden contribuir con una nueva perspectiva sobre la distribución de bosques de Polylepis y otras especies andinas con regímenes climáticos estrechos y distinguir las áreas de prioridad para su conservación. El objetivo de este estudio fue modelar la distribución potencial de la amplia gama Polylepis sericea y el vulnerable $P$. weberbaueri utilizando datos de clima y sensores remotos de alta resolución (1 km.). Este estudio también identificará el alcance geográfico de áreas protegidas que contienen estos bosques.

\section{Métodos}

Especies enfocadas y localización.- Las dos especies de Polylepis de este estudio tienen distintos rangos y ocupan diferentes hábitats en la Cordillera Andina. Polylepis sericea (Wedd.) tiene la más amplia distribución del género con una gama geográfica conocida desde Venezuela a Bolivia (Kessler 1995) muchas veces formando bosques húmedos de montaña. Habita en una variedad de ecosistemas de altitud desde el páramo al norte del Ecuador hasta regiones húmedas y tibias de Bolivia, el cual tiene temporadas más definidas. Polylepis weberbaueri (Pilg.) aparece desde el centro hasta el suroeste de Ecuador, en el noreste de Perú y poblaciones aisladas más al sur de la Cordillera Blanca y la zona de Cuzco-Apurimac. También es considerada vulnerable debido a una reducción en el tamaño de la población de $20 \%$ en los últimos 10 años debido a fragmentación y la recolección de madera (IUCN 2011).

En el presente estudio, las observaciones geográficas (localidades) representan la presencia observada de una especie. Para mejorar la precisión, este estudio escogió las localidades para $P$. sericea $(\mathrm{N}=85$, Fig. $2 \mathrm{~A})$ y P. weberbaueri $(\mathrm{N}=82$, Fig. $3 \mathrm{~A})$ del Missouri Botanical Garden's VAST (VAScular Tropicos) base de datos de nomenclatura (http://mobot.mobot.org/W3T/Search/ vast.htm) y Fjeldså y Kessler (2004), que reunió y verificó las localidades dentro de los pasados quince años para evitar áreas que se puedan haber deforestado. Esto también asegura que las localidades fueron seleccionadas durante la adquisición de los datos de sensores remotos utilizados en nuestra investigación. Antes de modelado, los puntos fueran superpuesta sobre las imágenes de Google Earth (versión 4.2) para verificar la exactitud de cada localización geográfica, basado en el las descripciones de la fuente (por ejemplo país, departamento, altitud, proximidad al punto de referencia, etc.) y la visibilidad los imágenes de moderada y alta resolución.

Capas ambientales.- Las capas ambientales contenían ambas capas de clima y ambientes remotamente sensoreadas producidas en los pasados seis años, el cual cubre Sudamérica en una resolución de $1 \mathrm{~km}$ (Tabla 2). Las capas del clima consisten en una

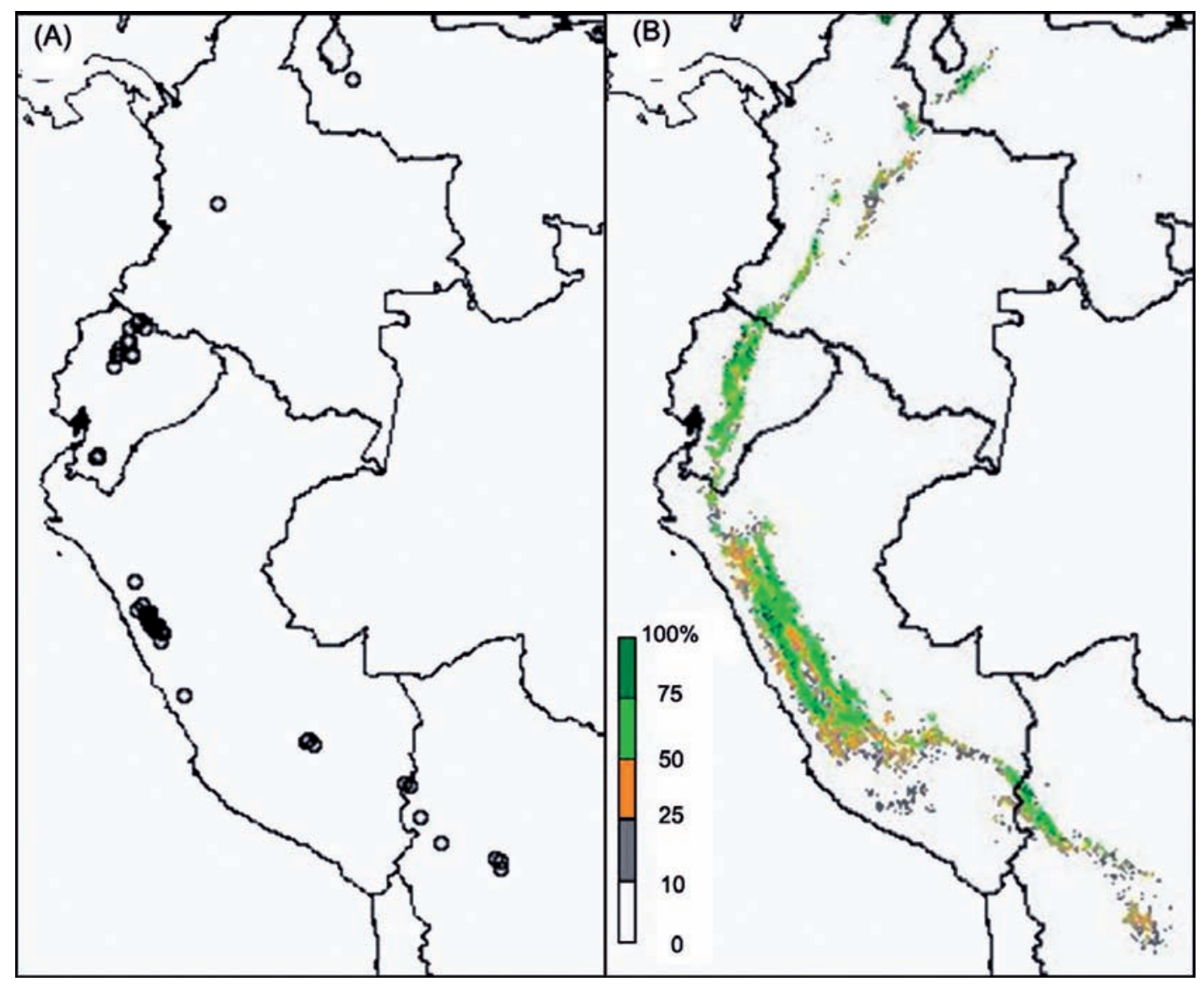

Figura 2. (A) Las localidades para $P$. sericea $(\mathrm{N}$ = 85) desde Venezuela hasta Bolivia y (B) distribución potencial. 


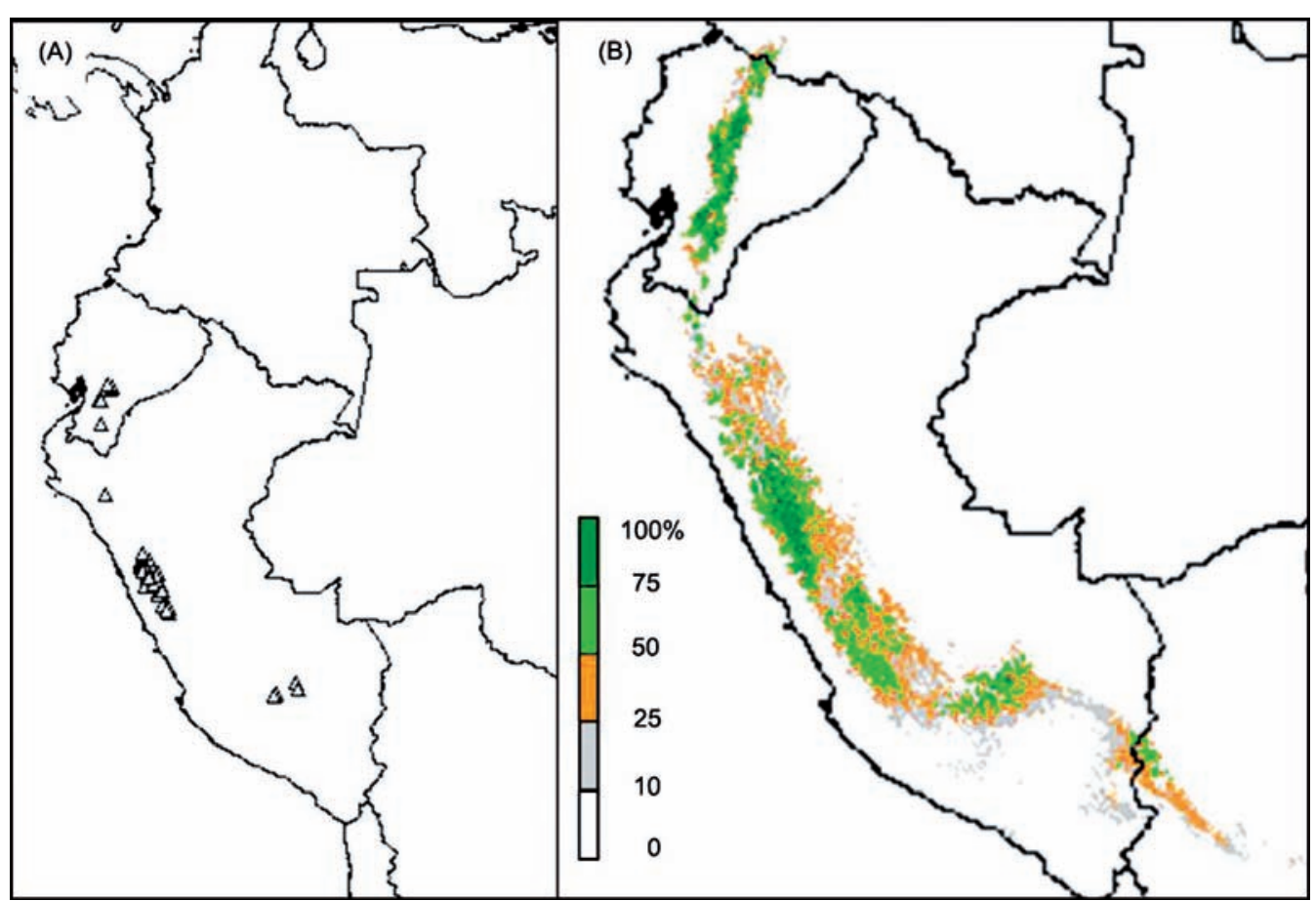

Figura 3. (A) Las localidades para $P$. weberbaueri $(\mathrm{N}=82)$ desde Ecuador y poblaciones aisladas en Perú y (B) distribución potencial.

serie de variables bioclimáticas, conocidas como Bioclim, obtenidas de la base de datos WorldClim (http://www.worldclim. org; Hijmans et al. 2005). Los datos de Bioclim representan las tendencias anuales de temperatura y precipitación producidas de un conjunto mundial de datos de estaciones de clima entre los años 1950-2000.

Las capas ambientales remotamente sensoreadas consisten en mediciones ópticas, microondas, y radares que miden una gama diversa de variables ambientales. Se utilizó el producto global de TERRA MODIS LAI (MOD15A2) de resolución de $1 \mathrm{~km}$ (Myneni et al. 2002). Compuestos mensuales (32 días) del Índice de Área de Hoja (LAI) de cuatro años (2001-2004) fueron formados para reducir los efectos de nubes y otros disturbios atmosféricos. La cobertura porcentual del árbol $(\sim 1 \mathrm{~km}$.) del año 2001 del MODIS vegetation continuous field (VCF), con una resolución nativa de $500 \mathrm{~m}$, fue también utilizados y puede servir como un indicador de la distribución espacial de la densidad de la biomasa (Hansen et al. 2002). Se incluyeron los datos globales del sensor de microonda QSCAT (Quick Scatterometer) disponibles en compuestos de 3 días en 2001 en una resolución de $2,25 \mathrm{~km}$. Las medidas son sensibles al contenido de agua en la hoja, la humedad, y aspereza de la superficie (Kunz \& Long 2005). Las medidas topográficas, promedio de elevación y la desviación estándar, se derivaron de los datos digitales de la elevación de SRTM, aumentadas desde 90 m a $1 \mathrm{~km}$. de resolución.

Después de la reducción de datos para llegar al mínimo de covarianza y facilitar la interpretación de la distribución de las especies (ver Buermann et al. 2008), un total de 18 capas del medio ambiente, en una resolución de $1 \mathrm{~km}$ espacial, se utilizaron inicialmente para este análisis. El subconjunto final de 11 capas bioclimáticas incluyen la temperatura media anual, promedio de temperatura diurna, estacionalidad de la temperatura, la temperatura máxima del mes más caluroso, la temperatura mínima del mes más frío, la precipitación anual, la estacionalidad de precipitación, la precipitación de los trimestres más húmedos, las precipitaciones de los trimestres más secos, la precipitación del trimestres más cálido, y la precipitación de los trimestres más fríos. El subconjunto de cinco capas de sensores remotos incluye el máximo anual LAI, el rango anual de LAI, el promedio anual de QSCAT y la desviación estándar de QSCAT (estacionalidad), y el porcentaje de cobertura arbórea. Las dos capas de topografía del SRTM (altura media y desviación estándar) también fueron incluidos.

Modelación.- Nuestra investigación requirió el uso del programa de Maxent (versión 3.2.1), el cual utilizó un algoritmo de máxima entropía para modelar la distribución de la especie, encontrando la distribución más cercana a una distribución

Tabla 2. Datos ambientales usados para el modelo de distribuciones potenciales de $P$. sericea y $P$. weberbaueri.

\begin{tabular}{llll}
\hline Datos & Variable ecológica & Fuente & Resolución \\
\hline Bioclim & $\begin{array}{l}\text { Variables derivadas mensualmente de temperatura y } \\
\text { precipitaciones } \\
\text { Elevación digital }\end{array}$ & WorldClim & $1 \mathrm{~km}$, años $\sim 1950-2000$ \\
Topografía y rugosidad & SRTM & $30 \mathrm{~m}$ \\
Porcentaje de cobertura arbórea & $\begin{array}{l}\text { Productividad neta, estacionalidad, la densidad de la } \\
\text { vegetación }\end{array}$ & MODIS & $1 \mathrm{~km}$, cada 8 días \\
Dispersómetro-retrodispersión & Rugosidad, la estacionalidad, y humedad de la superficie & QSCAT & 2,25 Km, cada 3 días \\
\hline
\end{tabular}


Tabla 3. Área estimada $\left(\mathrm{Km}^{2}\right)$ para $P$. sericea y $P$. weberbaueri y dentro de las áreas protegidas (AP) para países Andinos usando una estimado conservativo.

\begin{tabular}{|c|c|c|c|c|c|c|}
\hline \multirow{2}{*}{ Especies } & \multicolumn{6}{|c|}{ Área estimada $\left(\mathrm{Km}^{2}\right)$} \\
\hline & Ven & Col & Ecu & Per & Bol & Total \\
\hline P. sericea & 3,610 & 10,324 & 24,007 & 72,014 & 10,171 & 120,126 \\
\hline$(\mathrm{AP})$ & 2,395 & 3,103 & 5,020 & 8,932 & 3,498 & 22,948 \\
\hline P. weberbaueri & $3,444^{*}$ & $1,628^{*}$ & $26,547^{*}$ & 74,482 & 2,145 & 108,246 \\
\hline$(\mathrm{AP})$ & 2,262 & - & 3,894 & 6,690 & 1,692 & 14,538 \\
\hline
\end{tabular}

* Poblaciones nativas no existen en el país predicho.

uniforme a través del área del estudio (Philips et al. 2006). MAXENT proporciona un rango continuo de probabilidad a través del área geográfica de 0 a 1,0, dónde los valores altos indican la oportunidad relativamente más alta. En este estudio, los píxeles con valores iguales o por encima de 0,5 se identifican como áreas con una mayor probabilidad de presencia porque contienen las condiciones bioclimáticas típicas del nicho de una especie.

El subconjunto final de once bioclimas y cinco capas de sensores remotos con todas las localidades, se utilizaron para construir los modelos de distribución de las especies. Siguiendo a Phillips et al. (2006), hicimos 10 modelos para cada especie, seleccionando al azar $70 \%$ de los puntos para el modelo de capacitación y 30\% para las pruebas de modelo. El área bajo la curva ROC (Receiver Operating Characteristic) (AUC) proporcionan una medida del rendimiento del modelo. Un valor de AUC para un modelo puede variar de 0,5 (al azar) a 1,0 (discriminación perfecta). Es una pregunta importante que el uso general de datos de herbario y la posibilidad de sesgando los resultados puede afectar los resultados. Sin embargo, el efecto general con Maxent es limitado (Costa et al. 2010) y usamos Google Earth como otro nivel de verificación y eliminamos puntos que estaban en alturas no razonables.

\section{Resultados}

Los valores de AUC fueron estadísticamente significativos ( $\mathrm{P}<0,001$, Mann-Whitney U-Test) para todas las predicciones de distribución de las especies de Polylepis, lo que indica que todos los escenarios del modelo y las particiones de datos fueron significativamente mejores que el azar. El valor medio de las AUC, basado en 10 particiones al azar, para los modelos con todas las capas del medio ambiente fue $0,996 \pm 0,003$ para $P$. sericea y $0,994 \pm 0,002$ para $P$. weberbaueri.

La distribución prevista de $P$. sericea se refleja con la amplia distribución en los Andes tropicales del oeste de Venezuela hasta el norte de Bolivia (Fig. 2B). El promedio de elevación fue $3937 \pm 53 \mathrm{~m}$, una precipitación total anual de $927 \pm 32 \mathrm{~mm}$ y un promedio de temperatura mínima de $-2,2 \pm 0,5^{\circ} \mathrm{C}$. Casi el $60 \%$ de los bosques se prevé que se encuentra en el Perú (Tabla 3) la mayoría dentro de la Cordillera Blanca y Cordillera Huayhuash y la Cordillera Occidental y Oriental de los Andes del Perú central. Ecuador contiene 20\% de la extensión total, sobre todo en las cordilleras Occidentales y Orientales.

Alrededor del 93\% de P. weberbaueri fue previsto que ocurriera en el Ecuador y el Perú, con casi el 70\% en los Andes centrales del Perú, particularmente en la Cordillera Blanca y Cordillera Huayhuash (Fig. 3B). En el Perú, la elevación media fue $4056 \pm 3 \mathrm{~m}$, una precipitación total anual de $745 \pm 1 \mathrm{~mm}$ y un promedio de temperatura mínima de $-4,4 \pm 0,02{ }^{\circ} \mathrm{C}$. Del mismo modo, la altura media de estos bosques en el Ecuador fue $3481 \pm 4 \mathrm{~m}$, con una precipitación total anual de $861 \pm$
$2 \mathrm{~mm}$ y un promedio de temperatura mínima de 3,4 $\pm 0,03$ ${ }^{\circ} \mathrm{C}$. Pequeñas bolsas de hábitats fue provisto en regiones aisladas de Bolivia, Colombia y Venezuela pero estos representan menos del $4 \%$ del total de bosques potenciales.

La extensión de las áreas protegidas previstas $P$. sericea y $P$. weberbaueri son variables según el país. Áreas protegidas de Perú serían $8932 \mathrm{~km}^{2}$ a una altura promedio de $3400 \mathrm{~m}$ y $6700 \mathrm{~km}^{2}$ a una altura promedio de $2600 \mathrm{~m}$ para $P$. sericea y $P$. weberbaueri, sin embargo, esto sólo representa alrededor del 12\% y $9 \%$ de los bosques protegidos, respectivamente. La áreas protegidas en los Andes Venezolanos, se prevé que incluyen alrededor del 66\% de los bosques de P. sericea a una altura promedio de $5200 \mathrm{~m}$ y en Bolivia incluiría aproximadamente el $79 \%$ de $P$. weberbaueri. Esto está en agudo contraste con el total de bosques previstos para ser protegidas a lo largo de los Andes de alrededor del 19\% de $P$. sericea y el $13 \%$ de $P$. weberbaueri.

\section{Discusión}

Modelación y actual distribución de Polylepis. - La predicción de la distribución de la vegetación de altura, en particular los esenciales para la biodiversidad y la sostenibilidad, será cada vez más importante para esfuerzos de conservación en América del Sur. El manejo y restauración de bosques de Polylepis tiene un impacto directo en numerosas plantas endémicas y especies asociadas (Purcell et al. 2004), y la sostenibilidad de las poblaciones humanas locales dependiendo en servicios de los ecosistemas, incluido el mantenimiento de la hidrología y de la intercepción de neblina. La eficacia de los modelos de distribución de las especies proviene de la capacidad de utilizar un número limitado de observaciones con datos ambientales disponible al público para predecir rápidamente las especies locales o hábitats adecuados.

Las discrepancias entre los hábitats modelados y observados, probablemente como es el caso de Polylepis en este estudio, muchas veces son el resultado de la intensiva presión antropogénica histórica y moderna o limitada capacidad de dispersión de los hábitats disjuntos. Se cree que el Perú y Bolivia en la actualidad contienen menos del 3\% y $1 \%$, respectivamente, de la extensión histórica de los bosques de Polylepis (Fjeldså \& Kessler 1996), que nuestros modelos de predicción del potencial de una extensión forestal más grande de dos especies. La tasa de deforestación de bosques de Polylepis varía según la región. El 1\% se pierde en un plazo de 50 años en la Cordillera de Vilcanota, Perú (Jameson \& Ramsay 2007) hasta múltiples fragmentos que se talan en menos de 12 años en el noreste de los Andes Bolivianos (Purcell \& Brelsford 2004). Polylepis weberbaueri es conocida por las poblaciones disjuntas en Ecuador y Perú, y la distribución de modelado se extendió más allá de su área de distribución conocida hasta pequeños bolsones en Colombia y Venezuela. Clasificado como especie vulnerable por la UICN (2011), la presión antropogénica sería la razón principal para 
la situación corriente de poblaciones disyuntas, que incluyen grandes desconexiones entre poblaciones de P. weberbaueri.

Otra posibilidad para muchas poblaciones disyuntas de Polylepis son trasplantes contemporáneos e históricos. Nuestros modelos indican los bolsones de los hábitats adecuados fuera de las áreas conocidas por $P$. sericea y $P$. weberbaueri que reflejan el uso de Polylepis no nativos para la reforestación en diferentes regiones de los Andes. Trasplantes históricos, que han sobrevivido y se reproducen con éxito, puede haber causado poblaciones atípicas (Kessler \& Schmidt-Lebuhn 2006) y puede contribuir a la frecuente ocurrencia de hibridación entre especies a pesar de su aislamiento (Schmidt-Lebuhn et al. 2006, 2007). En un aspecto positivo, la facilidad de cultivo y trasplante de Polylepis (Fig. 4) permitiría una reforestación rápida y el aumento de la función hidrológica en regiones que se espera serán más secas con la pérdida de los glaciares.

Conservación y restauración de los bosques.- Tal vez los temas de importancia en materia de conservación en los Andes es la ubicación de las áreas protegidas en toda la región. Áreas protegidas con reconocimiento nacional e internacional tienen diferentes grados de alcance y eficacia, con un número mucho menor que ocurren en ambientes de altura (Rodríguez \& Young 2000, Soutullo et al. 2008). A raíz de nuestros modelos de predicción para dos especies de altura, la ubicación actual de las áreas protegidas, en Venezuela, Colombia, Ecuador, Perú y Bolivia, incluirán alrededor de $9 \%$ a casi el $80 \%$ de hábitats de Polylepis en cada país. Esto es indicativo de la falta de protección estándar de ecosistemas de altura en toda la región andina. El alto endemismo y la creciente importancia de los bosques de Polylepis para conservar la función del ecosistema se deben seguir poniendo en relieve en esta región para la conservación en las cuestiones del cambio climático.

Independientemente del estado de conservación, hay que destacar que los fragmentos de bosques de todos los tamaños sirven como hábitat esencial para la diversidad de alta elevación. Incluso los pequeńos fragmentos con una superficie total de bosques de menos de 4,5 ha, dentro de la Cordillera Blanca del Perú, se ha demostrado que mantienen altos niveles de diversidad de aves similares a los fragmentos de más de 20 ha (Rios et al. 2011). Sin embargo, los fragmentos pequeños deben permanecer dentro de los $200 \mathrm{~m}$ de fragmentos más grandes con el fin de mantener la conexión funcional de especies de aves (Lloyd \& Marsden 2011). Los pastizales entre los fragmentos, un componente importante del total de la matriz boscosa, es también un conductor de las comunidades de Polylepis que utilizan múltiples hábitats (Lloyd 2008, Bellis et al. 2009) y deben ser incluidos en los esfuerzos de conservación que se centran en aumentar la diversidad local. Todas estas facetas destacan la calidad de los bosques como un factor importante en el apoyo de la mayor diversidad de especies (Ríos et al. 2011) y la retención de la función ecológica (Lloyd \& Marsden 2011). Esto también incluye la conservación o el aumento de la complejidad estructural de los bosques, que contienen una mezcla de la regeneración de los árboles maduros (Renison et al. 2011), lo que aumentaría la disponibilidad de nichos y los recursos naturales de las diferentes etapas de la sucesión.

El estado de la diversidad genética de las poblaciones de bosques es un factor pasado por alto e importante a considerar también. Como con la mayoría de las especies de árboles tropicales, la fragmentación de los bosques de Polylepis disminuyen significativamente la diversidad genética de las poblaciones a pesar de ser polinizada por el viento, que a menudo muestran una menor pérdida genética de la fragmentación (Hensen et al. 2012). Además, las poblaciones en las elevaciones más altas tienen menor diversidad genética que otras poblaciones (Aragundi et al. 2011, Hensen et al. 2012) y se ha encontrado que la diversidad genética en $P$. multijuga era aproximadamente la mitad que otro especies polinizadas por el viento (Quinteros-Casaverde et al. 2012). Esto es posiblemente la resulta de los cuellos de botella genéticos y efectos fundadores durante las migraciones de ladera del Holoceno o la corta duración de la temporada de cultivo y temperaturas más frías en las elevaciones más altas (Hensen et al. 2012). La baja diversidad genética en las elevaciones más altas tendrá un impacto directo sobre la viabilidad de las primeras poblaciones que migran con el cambio climático. Además, la reforestación con especies no nativas de Polylepis puede conducir a la pérdida local de la diversidad genética de la hibridación, como otras especies relacionadas se sabe que se hibridan con facilidad (Schmidt-Lebuhn et al. 2006). La buena noticia es que gran cantidad de flujo de genes entre ellas y dentro de muchas poblaciones de Polylepis existe (Schmidt-Lebuhn et al. 2006) y la variabilidad genética parece estar plenamente disponible

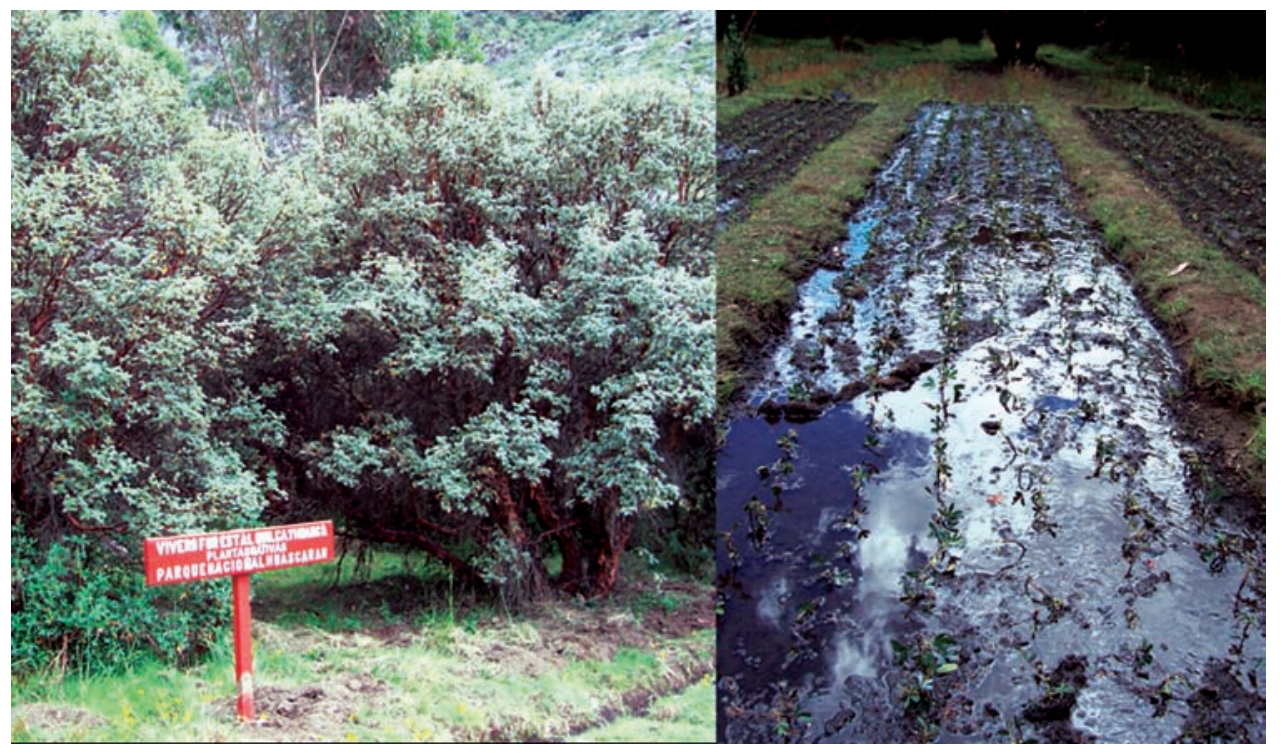

Figura 4. (A) Vivero forestal en la región de El Quilcayhuanca, Parque Nacional Huascarán, donde $P$. sericea y $P$. weberbaueri se cultivan para regenerar otros bosques de la región. (B) Parcelas de Polylepis plántulas. Fotos de Autor. 
para la restauración forestal en muchas áreas (Julio et al. 2011). Una posible estrategia es la recolección de semillas de múltiples fragmentos grandes y de individuos separados por al menos $25 \mathrm{~m}$ dentro de los fragmentos para maximizar la diversidad genética para reforestación (Quinteros-Casaverde et al. 2012).

\section{Conclusiones}

Hay varias implicaciones para futuras estrategias de conservación y restauración basadas en los modelos de distribución de dos especies de Polylepis que son mucho más grandes que la distribución actual. (1) Polylepis se puede restaurar a un área más amplia. Puede existir muchas áreas no disponibles para los bosques, debido a la agricultura u otros usos, pero fragmentos de Polylepis pequeños y continuos todavía pueden promover la biodiversidad y aumentar la función hidrológica de la región. (2) Las especies de Polylepis se pueden plantar en muchas zonas que son o no son nativas. El uso de poblaciones locales para la restauración de bosques sería preferible para evitar la hibridación y la pérdida de diversidad genética. (3) Nuevas áreas protegidas, privadas o públicas, pueden ser creadas específicamente para Polylepis, en lugares apropiados climáticamente y donde ahora no existen. Un plan estratégico con bosques de Polylepis puede incluir el uso sostenible de la biodiversidad de esta nuevo ecosistema, como el mercado limitado de orquídeas y otras plantas, y el ecoturismo, en particular la observación de aves, que puede ser conectados con un circuito de sitios arqueológicos para promover la conservación de ambos (Puhakka et al. 2011).

El reciente aumento en los estudios y la atención en los bosques de Polylepis reflejan la creciente importancia del mantenimiento de este ecosistema. Sin embargo, todavía quedan muchos espacios con respecto a nuestro conocimiento de las especies y la historia de la distribución de los bosques, la ubicación actual, y la respuesta al cambio climático. Modelización de la distribución actual y futura de todas las especies de Polylepis mejorará con la disponibilidad de los datos ambientales de alta resolución. Los modelos regionales del clima, producidas específicamente para predecir el cambio climático en el futuro de América del Sur, se espera que sean más precisos que los modelos de circulación global que ahora se utilizan para la región. Una alternativa importante para la adquisición de imágenes son las imágenes de alta resolución disponibles en Google Earth, que han sido utilizados para clasificar nuevos fragmentos de bosque en el surcentral del Perú (Benham et al. 2011) y representan un nuevo, libre y eficaz método de análisis de bosques de gran altitud. La susceptibilidad de estos bosques a una mayor fragmentación y la pérdida genética requieren una mayor protección y una planificación cuidadosa si la restauración es sostenible para todas las especies de Polylepis. Esperamos que la tecnología y técnicas avanzadas de manera significativa ayuden a mejorar el futuro de los bosques y las especies que dependen de ellos.

\section{Agradecimientos}

Agradecemos a Jorge Leal-Pinedo, Guillermo Velazco Zuta, Kiko Edson Roncal Velasco y Liliana Zuta de Roncal por la apreciable asistencia recibida durante los estudios de campo. Agradecer a los revisores anónimos por sus valiosos comentarios sobre versiones anteriores del manuscrito. Apreciamos también el apoyo de Juan Carlos Castro Vargas, ex-director del Parque Nacional Huascarán, Perú. Nos gustaría dar las gracias asimismo, al UCLA Latin America Center, UCLA Vavra fund, y al programa UC Pacific Rim el cual proporcionó financiamiento a Autor.

\section{Literatura citada}

Azócar A., F. Rada \& C. García-Núñez. 2007. Functional characteristics of the arborescent genus Polylepis along a latitudinal gradient in the high Andes. Interciencia 32: 663-668.

Bellis L.M., L. Rivera, N. Politi, E. Martín, M.L. Perasso, F. Cornell \& D. Renison. 2009. Latitudinal patterns of bird richness, diversity and abundance in Polylepis australis mountain forest of Argentina. Bird Conservation International 19: 265-276.

Benham P.M., E.J. Beckman, S.G. DuBay, L.M. Flores, A.B. Johnson M.J. Lelevier, C.J. Schmitt, N.A. Wright \& C.C. Witt. 2011. Satellite imagery reveals new critical habitat for endangered bird species in the high Andes of Peru. Endangered Species Research 13: 145-157.

Bourg N.A., W.J. McShea \& D.E. Gill. 2005. Putting the CART before the search: successful habitat prediction for a rare forest herb. Ecology 86: 2793-2804.

Buermann W., S. Saatchi, T.B. Smith, B.R. Zutta, J.A. Chaves, B. Milá \& C.H. Graham. 2008. Predicting species distribution across the Amazonian and Andean regions using remote sensing data. Journal of Biogeography 35: 1160-1176.

Cierjacks A., S. Salgado, K. Wesche \& I. Hensen. 2008. Post-fire population dynamics of two tree species in high-altitude Polylepis forests of Central Ecuador. Biotropica 40: 176-182.

Costa G.C., C. Nogueira, R.B. Machado \& G.R. Colli. 2010. Sampling bias and the use of ecological niche modeling in conservation planning: a field evaluation in a biodiversity hotspot. Biodiversity Conservation 19: 883-899.

Ellenberg H. 1979. Man's influence on tropical mountain ecosystems in South America. Journal of Ecology 67: 401-416.

Fjeldså J. \& M. Kessler. 1996. Conserving the biological diversity of Polylepis forests of the highlands of Peru and Bolivia. Nordic Foundation for Development and Ecology (NORDECO), Copenhagen.

Fjeldså, J. \& M. Kessler. 2004. Conservación de la biodiversidad de los bosques de Polylepis de las tierras altas de Bolivia: Una contribución al manejo sustentable en los Andes. Centro para la Investigación de la Diversidad Cultural y Biológica de las Bosques Pluviales Andinos (DIVA), Dinamarca.

Fjeldså J. 2002. Polylepis forests - vestiges of a vanishing ecosystem in the Andes. Ecotropica 8: 111-123.

García-Nuñez C., F. Rada, C. Boero, J. González, M. Gallardo, A. Azócar, M. Liberman-Cruz, M. Hilal \& F. Prado. 2004. Leaf gas exchange and water relations in Polylepis tarapacana at extreme altitudes in the Bolivian Andes. Photosynthetica 42: 133-138.

Guisan A. \& W. Thuiller. 2005. Predicting species distribution: offering more than simple habitat models. Ecological Letters 8: 993-1009

Hanselman J.A., W.D. Gosling, G.M. Paduano \& M.B. Bush. 2005. Contrasting pollen histories of MIS 5e and the Holocene from Lake Titicaca (Bolivia/Peru). Journal of Quaternary Science, 20: 663-670.

Hansen M.C., R.S. Defries, J.R.G. Townshend, R. Sohlberg, C. Dimiceli \& M. Carroll. 2002. Towards an operational MODIS continuous field of percent tree cover algorithm: examples using AVHRR and MODIS data. Remote Sensing of Environment 83: 303-319.

Hensen I., A. Cierjacks, H. Hirsch, M. Kessler, K. Romoleroux, D. Renison \& K. Wesche. 2012. Historic and recent fragmentation coupled with altitude affect the genetic population structure of one of the world's highest tropical tree line species. Global Ecology and Biogeography, 21: 455-464.

Hernandez P.A., I. Franke, S.K. Herzog, V. Pacheco, L. Paniagua, H.L. Quintana, A. Soto, J.J. Swenson, C. Tovar, T.H. Valqui, J. Vargas \& B.E. Young. 2008. Predicting species distributions in poorly studied landscapes. Biodiversity Conservation 17: 1353-1366. 
Hijmans R.J., S.E. Cameron, J.L. Parra, P.G. Jones \& A. Jarvis. 2005. Very high resolution interpolated climate surface for global land areas. International Journal of Climatology 25: 1965-1978.

IUCN. 2011. (en linea). 2011 International Union for Conservation of Nature (IUCN) Red List of Threatened Species, <http:// www.iucnredlist.org>. Accesso 07/03/2012.

Jameson J.S. \& P.M. Ramsay. 2007. Changes in high-altitude Polylepis forest cover and quality in the Cordillera de Vilcanota, Perú, 1956-2005. Biological Conservation 138: 38-46.

Julio N.B., J.C. Rondan Dueñas, D. Renison \& I. Hensen. 2011. Genetic structure and diversity of Polylepis australis (Rosaceae) tree populations from central Argentina: implications for forest conservation. Silvae Genetica 60: 55-61.

Kessler M. \& A.N. Schmidt-Lebuhn. 2006. Taxonomical and distributional notes on Polylepis (Rosaceae). Organisms, Diversity \& Evolution 6: 67-69.

Kessler M. 1995. The genus Polylepis (Rosaceae) in Bolivia. Candollea 50: 131-171.

Kessler M. 2002. The Polylepis problem: Where do we stand. Ecotropica 8: 97-110.

Kunz L.B. \& D.G. Long. 2005. Calibrating seawinds and QuikSCAT scatterometers using natural land targets. IEEE Geoscience and Remote Sensing Letters 2: 182-186.

Lloyd H. \& S.J. Marsden. 2011. Between-patch bird movements within a high-Andean Polylepis woodland/matrix landscape: Implications for habitat restoration. Restoration Ecology 19: 74-82.

Lloyd H. 2008. Abundance and patterns of rarity of Polylepis birds in the Cordillera Vilcanota, southern Perú: implications for habitat management strategies. Bird Conservation International 18: 164-180.

Mendoza W. \& A. Cano. 2011. Diversidad del género Polylepis (Rosaceae, Sanguisorbeae) en los Andes peruanos: Diversity of the genus Polylepis (Rosaceae, Sanguisorbeae) in the Peruvian Andes. Revista Peruana de Biología 18: 197-200.

Myneni R.B., S. Hoffman, Y. Knyazikhin, J.L. Privette, J. Glassy, Y. Tian, Y. Wang, X. Song, Y. Zhang, G.R. Smith, A. Lotsch, M. Friedl, J.T. Morisette, P. Votava, R.R. Nemani \& S.W. Running. 2002. Global products of vegetation leaf area and fraction absorbed PAR from year one of MODIS data. Remote Sensing of Environment 83: 214-231.

Philips S.J., R.P. Anderson \& R.E. Schapire. 2006. Maximum entropy modeling of species geographic distributions. Ecological Modeling 190: 231-259.

Puhakka L., M. Salo \& I.E. Sääksjärvi. 2011. Bird diversity, birdwatching tourism and conservation in Peru: a geographic analysis. PLoS ONE 6: e26786.doi:10.1371/journal. pone.0026786.

Purcell J. \& A. Brelsford. 2004. Reassessing the causes of decline of Polylepis, a tropical subalpine forest. Ecotropica 10: 155-158.

Purcell J., A. Brelsford \& M. Kessler. 2004. The world’s highest forest. American Scientist 92: 454-461.

Quinteros-Casaverde N., C.F. Flores-Negrón \& D.A. Williams. 2012. Low genetic diversity and fragmentation effects in a wind-pollinated tree, Polylepis multijuga Plige (Rosaceae) in the high Andes. Conservation Genetics 13: 593-603.
Raxworthy C.J., E. Martinez-Meyer, N. Horning, R.A. Nussbaum, G.E. Schneider, M.A. Ortega-Huerta \& A.T. Peterson. 2003. Predicting distribution of known and unknown reptil especies in Madagascar. Nature 426: 837-841.

Renison D., I. Hensen \& R. Suarez. 2011. Landscape structural complexity of high-mountain Polylepis australis forests: a new aspect of restoration goals. Restoration Ecology 19: 390-398.

Riordan E.C. \& P.W. Rundel. 2009. Modeling the distribution of a threatened habitat: the California sage scrub. Journal of Biogeography 36: 2176-2188.

Rios S.S, H. Lloyd \& A. Valdés-Velásquez. 2011. Bird species richness, diversity and abundante in Polylepis woodlands, Huascaran biosphere reserve, Peru. Studies on Neotropical Fauna and Environment 46: 69-76.

Rodriguez L.O. \& K.R. Young. 2000. Biological diversity of Peru: determining priority areas for conservation. AMBIO 29: 329-337.

Schmidt-Lebuhn A.N., M. Kessler \& M. Kumar. 2006. Promiscuity in the Andes: species relationships in Polylepis (Rosaceae, Sanguisorbeae) based on AFLP and morphology. Systematic Botany 31: 547-559.

Schmidt-Lebuhn A.N., M. Kumar \& M. Kessler. 2006. An assessment of the genetic population structure of two species of Polylepis Ruiz \& Pav. (Rosaceae) in the Chilean Andes. Flora 201: 317-325.

Schmidt-Lebuhn A.N., P. Seltmann \& M. Kessler. 2007. Consequences of the pollination system on genetic structure and pattern of species distribution in the Andean genus Polylepis (Rosaceae): a comparative study. Plant Systematics and Evolution 266: 91-103.

Servat G.P., C.W. Mendoza \& J.A. Ochoa C. 2002. Flora y fauna de cuatro bosques de Polylepis (Rosaceae) en la Cordillera del Vilcanota (Cusco, Peru). Ecología Aplicada 1: 25-35.

Simpson B.B. 1986. Speciation and specialization of Polylepis in the Andes. In: F. Vuillemeir and M. Monastrio, eds. High Altitude Tropical Biogeography, Oxford University Press, Oxford. Pp. 304-315.

Soutullo A., M. DeCastro \& V. Urios. 2008. Linking political and scientifically derived targets for global biodiversity conservation: implications for the expansion of the global network of protected areas. Diversity and Distribution 14:604-613.

Teich I., A.M. Cingolani, D. Renison, I. Hensen \& M.A. Giorgis. 2005. Do domestic herbivores retard Polylepis australis Bitt. woodland recovery in the mountains of Córdoba, Argentina? Forest Ecology and Management 219: 229-241.

Thomassen H.A., T. Fuller, W. Buermann, B. Milá, C.M. Kieswetter, P. Jarrín-V., S.E. Cameron, E. Mason, R. Schweizer, J. Schlunegger, J. Chan, O. Wang, M. Peralvo, C.J. Schneider, C.H. Graham, J.P. Pollinger, S. Saatchi, R.K. Wayne \& T.B. Smith. 2011. Mapping evolutionary process: a mutlitaxa approach to conservation prioritization. Evolutionary Applications 4: 397-413.

Wilson K.A., M.I. Westphal, H.P. Possingham \& J. Elith. 2005. Sensitivity of conservation planning to different apporoaches to using predicted species distributions on the east slope of the Andes in Peru and Bolivia. Biological Conservation 122: 99-112. 